\title{
¿Eficiencia o suficiencia \\ en el sector de gestión privada?
}

\section{Artículo de reflexión $\quad \begin{aligned} & \text { de Educación, } \mathrm{N}^{\circ} 70 . \\ & \text { Primer semestre de } 2016\end{aligned}$ \\ Bogotá, Colombia.}

//Efficiency or Sufficiency
in the Private Management Sector?

//Eficiência ou suficiência no

setor de gestão privada?
Silvia Montoya*
María Laura Frugoni**
Recibido: 29/07/2015 Evaluado: 29/09/2015

Silvia Montoya*
María Laura Frugoni**

\footnotetext{
* Doctora en Análisis de Políticas en la Pardee RAND Graduate School y magister en Administración Pública de la Kennedy School of Government, Harvard University. Directora del Instituto de Estadísticas de la Unesco. Montreal, Canadá. Correo electrónico: s.montoya@unesco.org

* $\quad$ Licenciada en Economía de la Pontificia Universidad Católica de Argentina. Miembro del grupo de trabajo de la web institucional Provincia NET. Correo electrónico: maiafrugoni@gmail.com
}

\section{Resumen}

Esta investigación gira en torno a una pregunta central: ¿es la educación de gestión privada más eficiente que la de gestión pública? En primer lugar, se define a la "eficiencia técnica" como el máximo nivel de producto (ej: nivel de aprendizajes) con una cantidad de insumos dada (ej: habilidades del docente, tamaño del aula, etc). Este enfoque en la relación entre inversión y resultados divide el ejercicio comparativo en múltiples aristas. Sobre la base de información obtenida de evaluaciones e índices elaborados para la Cuidad Autónoma de Buenos Aires, se realiza un recorrido por todas estas dimensiones ¿Cuál es la importancia, participación, proporción de alumnos que reciben y el rol de las escuelas de gestión privada? ¿Cómo es la estructura de financiamiento? ¿En qué medida la educación privada depende de fondos estatales o aportes de los padres? ¿Con qué recursos totales disponen los establecimientos de cada tipo de gestión? ¿Cómo se distribuyen los fondos? ¿Qué niveles de calidad educativa obtienen? ¿Cuánto influye en los resultados el nivel socioeconómico de los alumnos o el contexto económico, demográfico o geográfico? ¿Qué resultados de aprendizaje, trayectoria y niveles de equidad obtienen las escuelas según su tipo de gestión? Todas estas respuestas terminan reformulando la pregunta del inicio: ¿qué resultados de aprendizaje se obtendrían en cada sector de gestión con niveles equivalentes de gasto y una población escolar en las mismas condiciones socioeconómicas?

\section{Abstract}

This research focuses around the question: Is privately managed education more efficient than publicly managed education? To begin, 'technical efficiency' is defined as the highest level of product (learning level) achieved with the supplies available (teacher skills, classroom size, etc). The focus on the relationship between investment and results makes the comparison a task to be addressed from different approaches. A list of all these aspects has been made based on information obtained from evaluations and research for the Autonomous city of Buenos Aires. What is the importance, involvement, number of students enrolled, and the role played by privately managed schools? What is the financing structure? To what measure does private education depend on the State's funding, or contributions made by families? What is the total amount of economic resources assigned to each type of management? How are funds

\section{Palabras clave}

Equidad, eficiencia técnica, calidad educativa, financiamiento, distribución, factor socioeconómico, sectores de gestión

\section{Keywords}

Equity, efficiency, quality of education, financing, allocation, socioeconomic factor, management sectors

\section{Palavras chave}

Equidade, Eficiência técnica, Qualidade educativa, Financiamento, Distribuição, Fator socioeconômico (Setores de gestão) 
allocated? What is the quality of the education provided? What's the influence of the students' socioeconomic level, and economic, demographic or geographic background on their progress? What results in terms of learning, trajectory, and equity levels does a school obtain under each type of management? The answers to these questions lead to restating the initial question: What learning outcomes would be obtained under each management sector with equivalent spending levels and enrollment under the same socioeconomic conditions?

\section{Resumo}

A pesquisa gira em torno a uma pergunta central: É a educação de gestão particular mais eficiente que da gestão pública? Em primeiro lugar, define-se à "eficiência técnica" como o máximo nível de produto (ex.: nível da aprendizagem) com uma quantidade de insumos dada (ex.: habilidades do docente, tamanho da aula, etc.). Esse enfoque, na relação entre inversão e resultados, divide o exercício comparativo em várias arestas. Sob a base de informação obtida de avaliações e índices elaborados para a Cidade Autônoma de Buenos Aires, se realiza um percorrido por todas as seguintes dimensões: Qual é a importância, participação, proporção de alunos que recebem a função das escolas de gestão particular? Como é a estrutura de financiamento? Em que medida a educação particular depende de fundos estatais ou aportes dos pais? Com que recursos totais dispõem os estabelecimentos de cada tipo de gestão? Como se distribuem os fundos? Que níveis de qualidade educativa obtém? Quanto influi nos resultados o nível socioeconômico dos alunos ou o contexto econômico, demográfico ou geográfico? Que resultados de aprendizagem, trajetória e níveis de equidade obtém as escolas segundo seu tipo de gestão? Todas estas respostas terminam reformulando a pergunta do início: Que resultados de aprendizagem se obteriam em cada setor de gestão com níveis equivalentes de gasto, e uma povoação escolar nas mesmas condições socioeconômicas?

La participación y el rol de la educación de gestión privada, la cobertura en cuanto a matrícula, financiamiento y eficiencia son temas que están omnipresentes en el debate educativo desde hace décadas.

Existen distintas teorías acerca del rol y la importancia de la educación privada. Friedman sostenía que los padres deberían recibir vouchers, de manera que pudieran elegir enviar a sus hijos a escuelas de gestión privada, en lugar de a escuelas estatales; de esta manera le daba mayor relevancia a las escuelas privadas.

En contraposición, el estudio realizado por Somers et al. (2011) muestra cómo la mejora educativa de la escuela privada por encima de la estatal cae, e incluso llega a ser negativa en algunos casos, a medida que se va controlando por variables del contexto socioeconómico del alumno y las características de los compañeros de aula. 
Al hablar acerca de la distinción entre estatal y privada se suele destacar la segregación social. Según Narodowski y Andrada (2000), cada vez en mayor medida el sector de gestión estatal absorbe población de nivel socioeconómico bajo, mientras sucede lo contrario en el sector de gestión privada. Esta afirmación requeriría de mayor acción y soporte para el sector más vulnerable, y a su vez de mayor intervención para mejorar el acceso al sector de educación privada.

De esta manera, son diversos los temas que se discuten respecto a la educación de gestión privada: participación, financiamiento, prestaciones. Sin embargo, son dos los pilares de discusión centrales: equidad y eficiencia. ¿Cómo analizar la educación en un contexto en el cual los padres aportan dinero y compensan diferencias, en el cual los alumnos que asisten a cada tipo de escuela son diferentes y no es sencillo diferenciar lo que es eficiencia de lo que es ventaja comparativa por el tipo de alumnado?

Con este artículo se pretende contribuir a un mayor conocimiento de la situación educativa actual, teniendo como principales pilares de discusión la equidad y la eficiencia, y destacar si están siendo logradas y qué queda por mejorar para alcanzar una educación de calidad para todos los ciudadanos.

A través de un repaso del sistema de educación privada, siempre de la mano de los conceptos de equidad y eficiencia, se realizará una revisión para el caso de la Ciudad de Buenos Aires, abordando temas tales como el esquema de aportes estatales a escuelas de gestión privada y la inversión en educación.

\section{La eficiencia de la escuela privada}

En una primera perspectiva, parecería que el simple hecho de privatizar escuelas contribuiría a un sistema educativo más eficiente y efectivo. Sin embargo, igenera mejores resultados de aprendizaje? ¿Implica un menor costo?

El debate suele centrarse en este tipo de interrogantes a la hora de determinar si el sector de gestión privada genera eficiencia o ineficiencia, analizando a su vez la estructura del financiamiento del Estado a escuelas de este sector de gestión.

Según el estudio realizado para Chile por Carnoy y McEwan (1997), al hablar de eficiencia se distinguen dos definiciones. Por un lado, la eficiencia técnica: cuando se produce el máximo nivel de producto (por ejemplo, nivel de aprendizaje) con una cantidad de insumos dada (por ejemplo, docentes capacitados tamaño del aula, etc). Y por el otro, la eficiencia de asignación: implica una combinación óptima de insumos (escolares en este caso), en consideración de sus precios dados.

A lo largo del artículo se dimensiona la eficiencia técnica, analizando los costos asociados a los insumos educativos en cada sector de gestión y considerando la "calidad 
educativa" como su producto. Se trata de un producto compuesto, que se puede entender como el grado de alcance del conjunto de objetivos que se consideran primordiales para el sistema educativo. La eficiencia técnica es indisociable de la inteligencia de la estrategia para organizar los recursos disponibles según los fines perseguidos. Por lo tanto, la definición de "calidad educativa" implica un acuerdo sobre los fines del sistema educativo, en este caso la graduación de los estudiantes a tiempo y con los saberes mínimos necesarios para insertarse de manera productiva en la sociedad. Esta definición es justamente la que consideró la Dirección General de Evaluación de Calidad Educativa del Gobierno de la Ciudad de Buenos Aires al desarrollar su más elaborada herramienta de medición, el Índice de Equidad y Calidad de la

Educación Porteña (IECEP). El IECEP reúne en su fórmula ocho indicadores que integran aspectos referidos a logros de aprendizaje (rendimientos altos y medios en pruebas de Matemática y Lengua), trayectoria de los alumnos (según tasas de promoción y repetición) y equidad de los aprendizajes (comparando los resultados de cada escuela con los promedios de la ciudad). Siguiendo el razonamiento, la eficiencia técnica de un establecimiento educativo se concibe como la relación entre los insumos o montos invertidos y los resultados obtenidos en estos tres grandes aspectos señalados, expresados en el IECEP. Este enfoque permite medir y comparar la eficiencia promedio de los establecimientos según el tipo de gestión, entre muchas otras variables.

Las escuelas de gestión privada por lo general cuentan con una ventaja asociada a las características de la población que asiste a este sector de gestión: suelen situarse en zonas urbanas donde el nivel de capital humano es mayor, los padres de los alumnos suelen tener un nivel socioeconómico más alto, entre otras ventajas (Carnoy \& McEwan, 1997).

Por lo tanto, esta concepción teórica de la eficiencia debe salvar el hecho de que un alumno recién ingresado al sistema escolar no es un insumo homogéneo sino que proviene de entornos socioeconómicos desiguales. Está demostrado que estos aspectos contextuales inciden en los resultados de aprendizaje de los alumnos, tanto en la predisposición inicial como a lo largo del proceso, y generan una brecha positiva en favor de los alumnos en escuelas de gestión privada y una posible distorsión sobre la eficiencia y eficacia de las escuelas privadas. Para compensar estos factores en la medición se realizará también un ajuste a dicho índice por nivel socioeconómico (IECEPa). 
El financiamiento es otra área de debate relacionada. El esquema es bastante más complejo que el pago de arancel. Las escuelas de gestión estatal están manejadas por un criterio de financiamiento de la oferta con fondos que provienen del Estado. En el caso de la educación privada el financiamiento puede estar dado tanto por los padres de los alumnos, mediante el pago mensual de una cuota, como por el Estado a través de los aportes estatales que dependen justamente del nivel de la cuota. Se agregan en ambos casos los gastos de bolsillo de los padres. El esquema que adopte el Estado en su mecanismo de financiamiento y la efectiva implementación son esenciales para lograr mejoras en cuanto a equidad y eficiencia (Carnoy \& McEwan, 1997).

Medir el grado de equidad implica dilucidar en qué medida el contexto socioeconómico limita la eficiencia técnica o el alcance de los objetivos educativos. Hay condiciones sociales que afectan el desarrollo pleno del individuo, tanto al ingresar al sistema educativo como a lo largo del proceso y a su egreso. La propuesta es partir de una comparación del producto, dado que los resultados educativos que se encuentran por debajo del promedio de la ciudad, trayectorias demoradas o interrumpidas y rendimientos académicos insuficientes son en sí mismos inequidades presentes en la medida en que suponen una limitación para la posterior inserción productiva en la sociedad. El segundo paso es dilucidar el peso relativo que tuvo el contexto socioeconómico, o las inequidades pasadas, en estos bajos rendimientos, mediante una comparación geográfica y por tipo de gestión. Por último, esta información permite también evitar inequidades futuras mediante una distribución más equitativa del presupuesto para que Ileguen más fondos al rescate de zonas vulnerables. El enfoque es compatible con la definición de la Organización de las Naciones Unidas para la Educación, la Ciencia y la Cultura (Unesco), que define la equidad educativa como educar de acuerdo a las diferencias y necesidades individuales, para lograr que las condiciones económicas, demográficas, geográficas, étnicas o de género no supongan un impedimento para el aprendizaje.

Luego de esta breve presentación el trabajo se aproximará al análisis de eficiencia en el sector de gestión privada, partiendo de la estructura y magnitud de dicho sistema en Argentina, específicamente en la Ciudad Autónoma de Buenos Aires (CABA). La presentación del esquema de aportes estatales a escuelas de gestión privada y su funcionamiento en la práctica conformarán parte del análisis. Se estudiará la eficiencia en dicho sector de gestión a través de un sistema de microcostos para la CABA, ajustado primero por un indicador de calidad y equidad, y luego por un indicador del nivel socioeconómico. 


\section{Importancia y participación de la escuela privada en Argentina}

Hacia 1822, el sistema educativo argentino se caracterizaba por la preponderancia de las escuelas particulares o privadas. Mientras se contaba únicamente con 9 escuelas estatales de primeras letras, eran 55 las escuelas particulares ${ }^{1}$. Marcando desde hace dos siglos la importancia de la educación privada, el sistema educativo ha ido evolucionando al respecto.

La participación de la escuela privada fue creciendo desde ese entonces hasta la Ley 1420, de finales del siglo XIX, para luego comenzar a caer (Montoya et al., 2001). La descentralización educativa y la mayor autonomía otorgada a las escuelas de gestión privada fue generando a partir de la década de los sesenta un mayor crecimiento del sector de gestión privada (Narodowski \& Andrada, 2000).

Gráfico 1. Serie de matrícula de educación común de gestión privada y PB।

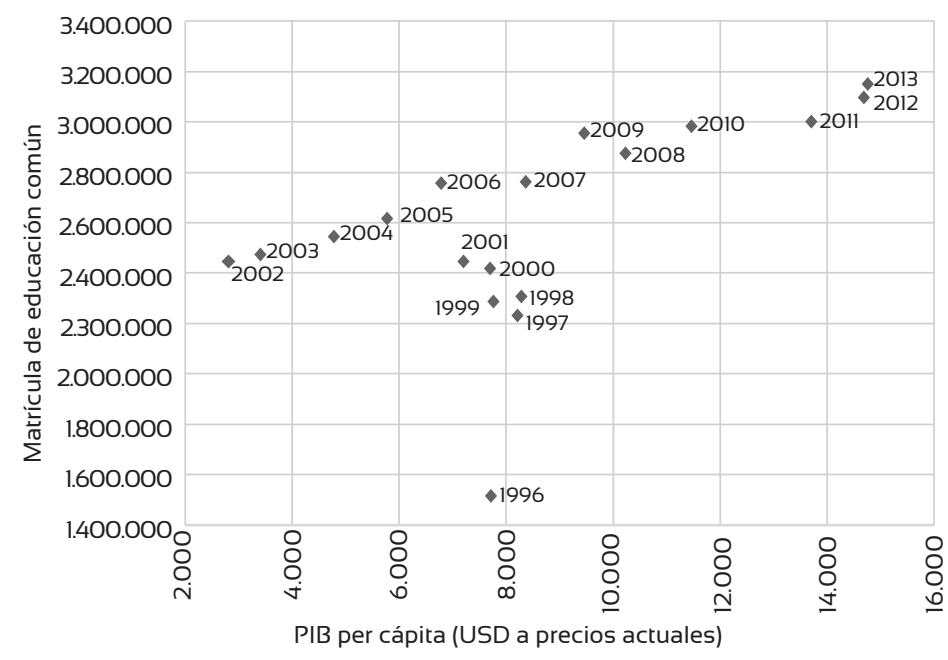

per cápita para Argentina, 1996-2013

Nota: Educación común incluye los niveles inicial, primario, secundario y superior.

Fuente: Elaboración propia con base en datos del Relevamiento Anual 1996-2013, DINIECE, Dirección Nacional de Información y Evaluación de la Calidad Educativa, Ministerio de Educación de la Nación, e Indicadores del Desarrollo Mundial (1996-2013) - Banco Mundial. 
Si bien las escuelas del sector de gestión estatal reciben actualmente la mayor proporción de alumnos $(66,7 \%$ en el nivel inicial, $75,3 \%$ en el nivel primario y $73,9 \%$ en el nivel secundario), son más de 3 millones los alumnos de Argentina que asisten a escuelas de gestión privada ${ }^{2}$.

La matrícula del sector de gestión privada ha ido evolucionando en las últimas décadas al igual que el producto bruto interno ( $\mathrm{PBI}$ ) per cápita. La tendencia a partir del 2000 en ambos casos fue creciente, con excepción de los años posteriores a la crisis argentina (2001) y la crisis de las hipotecas subprime (2008).La participación de las transferencias del Estado al sector de gestión privada dentro del gasto educativo total da muestras de la importancia asignada a este sector de gestión. Entre 2001 y 2013, tanto el gasto educativo total como las transferencias a la educación privada presentaron una tendencia creciente. La participación de las transferencias a la educación privada dentro del gasto total se mantuvo relativamente, oscilando entre valores del 12 y $13 \%$ como se puede observar en el gráfico 2.

Gráfico 2. Evolución del gasto total en educación y de las transferencias a la educación privada (en millones de pesos) para Argentina (2001-2013)

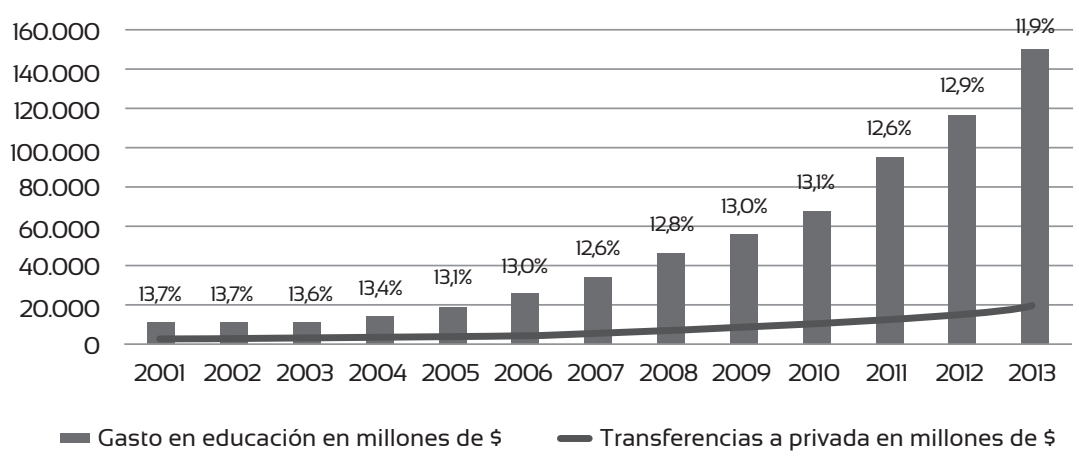

Nota: Incluye el gasto en educación a cargo de las provincias y de la Ciudad Autónoma de Buenos Aires. Fuente: Elaboración propia con base en datos de la Coordinación General de Estudio de Costos del Sistema Educativo, Dirección de Planeamiento Educativo, Ministerio de Educación de la Nación. 
En el caso particular de la Ciudad de Buenos Aires, la evolución del gasto y las transferencias a la educación privada también fue creciente y su participación mayor que a nivel país con valores entre 15 y $19 \%$ (gráfico 3).

Este trabajo se focalizará en el sistema educativo de la Ciudad Autónoma de Buenos Aires (CABA), específicamente en la educación común.

Gráfico 3. Evolución del gasto total en educación y de las transferencias a la educación privada (en millones de pesos) para la Ciudad Autónoma de Buenos Aires (2001-2013)

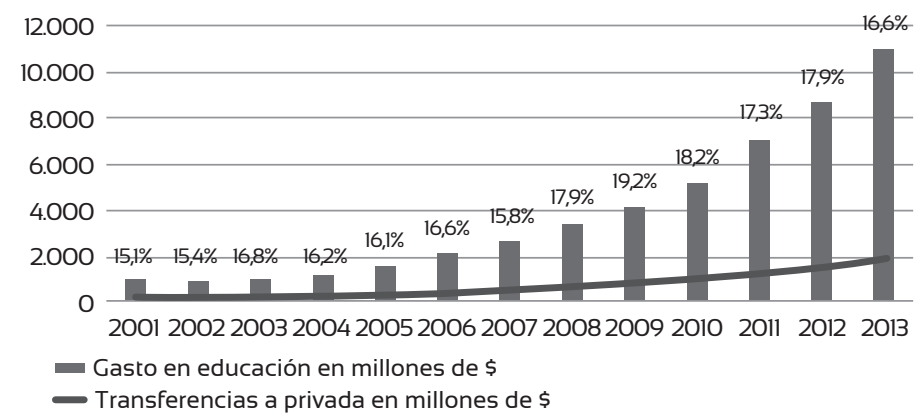

Fuente: Elaboración propia con base en datos de la Coordinación General de Estudio de Costos del Sistema Educativo, Dirección de Planeamiento Educativo, Ministerio de Educación de la Nación.

\section{El sistema educativo en la Ciudad Autónoma de Buenos Aires}

Como una de las 24 jurisdicciones de Argentina, la CABA cuenta con un sistema educativo gestionado y sustentado en su propio Ministerio de Educación.

La mayor proporción de matrícula que asiste a establecimientos educativos de la CABA se encuentra en la educación común. Dentro de la misma, el nivel educativo con mayor número de alumnos es el primario, le siguen el secundario y el inicial.

Si bien la matrícula del sector de gestión estatal es mayor que la del sector de gestión privada en los niveles primario y secundario, las proporciones son relativamente similares entre ambos sectores de gestión y la matrícula de gestión privada ha ido creciendo en la última década, como se puede observar en el gráfico 4 . A nivel país, la diferencia de matrícula según sector de gestión es notoria; la participación del sector de gestión estatal es muy superior a la del sector privado. 
Gráfico 4. Evolución de la matrícula inicial, primaria y secundaria común según sector de gestión. CABA (1996-2011)

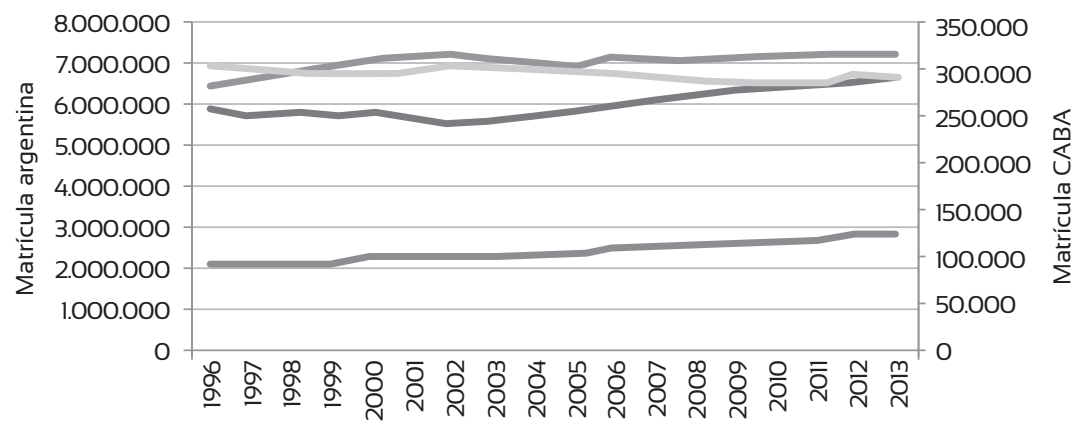

Estatal ARG Privada ARG $\longrightarrow$ Estatal CABA Privada CABA

Fuente: Elaboración propia con base en datos de la Gerencia Operativa de Investigación y Estadística, Unidad de Evaluación Integral de la Calidad y Equidad Educativa y a DiNiece, Ministerio de Educación de la Nación.

Gráfico 5. Número de alumnos del nivel primario de la escuela privada por cada alumno de la escuela estatal, por comuna. CABA (2014)

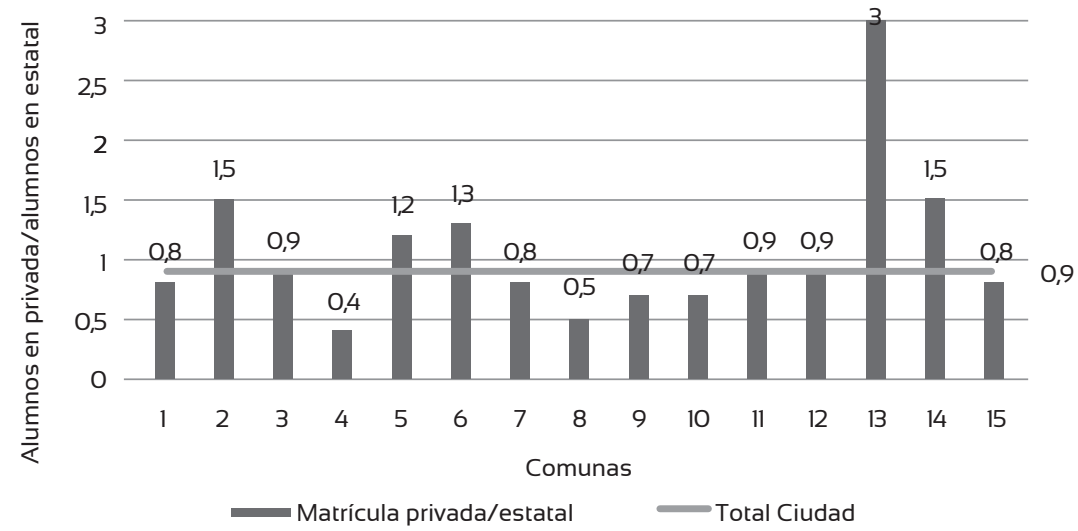

Fuente: Elaboración propia con base en datos de S. Montoya (2014).

Es notoria la presencia de la escuela privada dentro delnivel primario de la ciudad; sin embargo, existe una gran variabilidad entre las quince comunas en las cuales está dividida administrativamente.

La mayor proporción de niños y jóvenes en edad escolar (entre 5 y 17 años) se encuentra en la zona sur, que es la de menor nivel socioeconómico.
La geografía económica determina la geografía de la presencia de la gestión privada. Aproximadamente 1 de cada 3 alumnos que asisten a escuelas estatales es de la zona sur. En las comunas 13 y 14 es mayor la cantidad de alumnos del nivel primario que asiste a escuelas privadas que a estatales. Lo contrario sucede para las comunas de la zona sur (4, 8 y 9$)$. 
Cuadro 1. Matrícula según nivel educativo, sector de gestión y tipo de educación. CABA (2012)

\begin{tabular}{|l|l|l|l|r|}
\hline \multicolumn{1}{|c|}{$\begin{array}{c}\text { Tipo de } \\
\text { educación }\end{array}$} & $\begin{array}{c}\text { Nivel de } \\
\text { educación }\end{array}$ & \multicolumn{2}{|c|}{ Total } & \multicolumn{2}{c|}{ Sector de gestión } \\
\hline Total & Estatal & \multicolumn{1}{c|}{ Privado } \\
\hline Común + especial + adultos & 731.071 & 368.614 & 362.457 \\
\hline & Total & 680.876 & 328.072 & 352.804 \\
\hline & Inicial & 116.879 & 50.965 & 65.914 \\
\hline Común & Primario & 278.885 & 146.36 & 132.525 \\
\hline & Secundario & 181.302 & 92.376 & 88.926 \\
\hline Especial & Superior & 103.81 & 38.371 & 65.439 \\
\hline Adultos & Total & 4.913 & 2.838 & 2.075 \\
\hline Otros servicios educativos & Total & 45.282 & 37.704 & 7.578 \\
\hline
\end{tabular}

Fuente: Elaboración propia con base en datos del Relevamiento Anual 2012, Unidad de Evaluación Integral de la Calidad y Equidad Educativa (UEICEE), MEGC.

\section{Aportes estatales a escuelas de gestión privada}

Los subsidios a escuelas privadas se establecen como aportes que el Estado otorga a las escuelas de este sector para promover la igualdad de oportunidades y el derecho de toda persona a elegir el tipo de escuela al que desea acceder.

El Decreto Nacional 2542/91 sostiene que el objetivo de los aportes es "[...] asegurar la opción de los padres de elegir la escuela para sus hijos, en el marco de la libertad de enseñar y aprender que garantiza la Constitución Nacional [...]" (artículo 1. ).

El eje de discusión se centra en si la regulación existente deja espacio para la inequidad o logra solventar esta problemática, y si su funcionamiento en la práctica difiere de la teoría y la normativa.

La Unesco define la equidad en educación como educar de acuerdo a las diferencias y necesidades individuales, para lograr que las condiciones económicas, demográficas, geográficas, étnicas o de género no supongan un impedimento para el aprendizaje. 
El financiamiento público a la educación de gestión privada se da a través de la demanda, no de la oferta como en el caso chileno, y el grado de financiamiento debe estar en relación con las características socioeconómicas de la propia escuela para evitar la inequidad, según la definición anterior.

En el caso de los establecimientos educativos que perciben aranceles, el Decreto 2542/91 establece que la asignación del porcentaje de financiamiento se debe realizar con base en: 1) las características económicas y sociales de la zona y la población escolar; 2) el nivel educativo de la institución y la modalidad; 3) los resultados del balance de la situación financiera del establecimiento; 4) las necesidades del instituto como unidad escolar en su zona de influencia;
5) la categoría en la que solicite ser inscrito, el monto del arancel percibido por el establecimiento y los aranceles máximos establecidos por la Dirección General de Educación de Gestión Privada (DGEGP) y CABA.

\section{De la teoría a la práctica}

Enmarcada en la normativa vigente, la asignación de subsidios se constituye en una de las herramientas de política. Según los datos del 2012 para la CABA, las comunas con mayor porcentaje de escuelas primarias de gestión privada que reciben aportes estatales son las comunas 1, 4, 8 y 9. De estas, la 8 es la de menor proporción sin subsidio $(0 \%)$ y la 4 es la de mayor proporción con aportes del $100 \%(72 \%)$.

Gráfico 6. Distribución de establecimientos de educación primaria cada 100 de acuerdo al porcentaje de aportes que reciben, por comuna. CABA (2012)

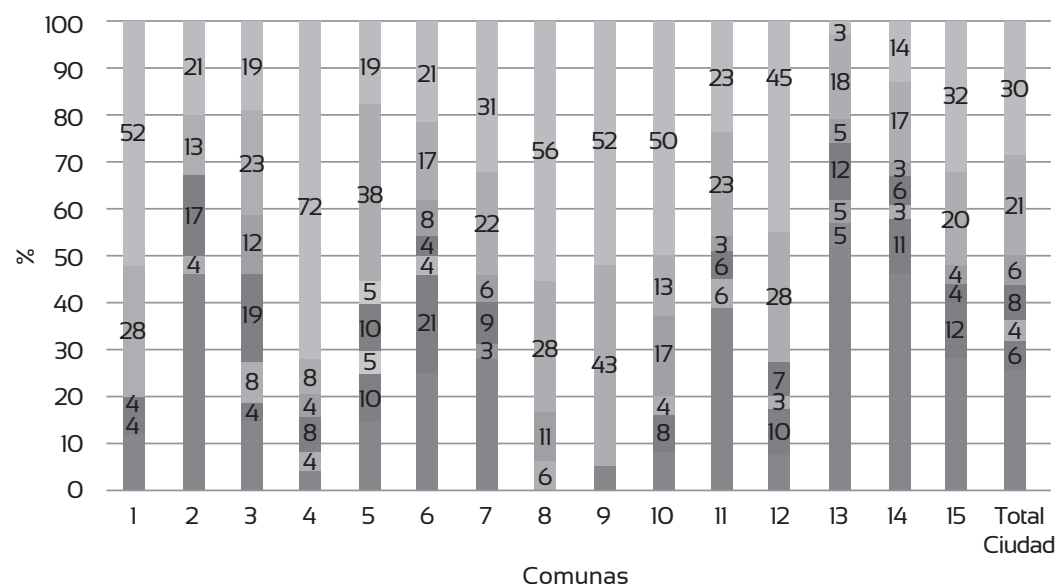

$\square 0 \quad \square 40 \quad \square 0 \quad \square 60 \quad \square 0 \quad-80 \quad \square 100$ 
Por otro lado, las comunas 2, 13 y 14 son las que cuentan con el mayor número de establecimientos educativos que no reciben aportes estatales.

Retomando el motivo detrás de la asignación de subsidios a escuelas privadas y el concepto de equidad deseado en la norma, las comunas que reciben mayores aportes estatales deberían ser las más vulnerables.

Al observar los valores del índice de vulnerabilidad social (IVS) ${ }^{3}$ para las comunas de la CABA se observa justamente que aquellas comunas con mayores valores del índice presentan un mayor porcentaje de escuelas privadas que reciben aportes estatales.

De esta manera, las comunas que son socioeconómicamente vulnerables cuentan con una mayor proporción de establecimientos educativos que reciben aportes. Por otro lado, las comunas menos vulnerables cuentan con una menor proporción de escuelas privadas subsidiadas.

Gráfico 7. Índice de vulnerabilidad social y porcentaje de escuelas primarias privadas que reciben aportes estatales, por comuna. CABA

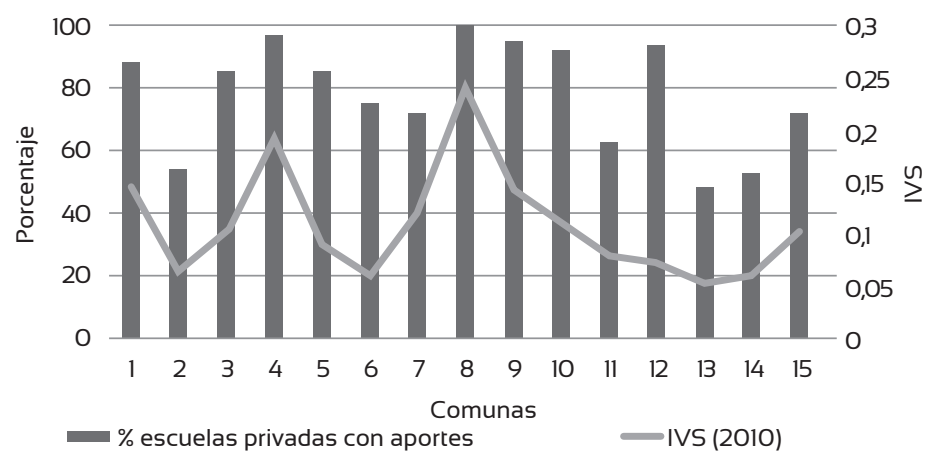

Fuente: Elaboración propia con base en datos de S. Montoya (2014).

3 El ivs mide características demográficas, económicas, habitacionales y de acceso a la salud y educación de los hogares. 


\section{Equidad y eficiencia según gestión}

En los presupuestos del Ministerio de Educación del Gobierno de la Ciudad (MEGC) se observa que si bien el monto destinado al sector educativo entre 2010 y 2014 se ha incrementado en términos absolutos, ha ido disminuyendo en términos relativos con respecto al gasto total del GCBA. La participación del gasto del MEGC dentro del gasto total del GCBA cayó de $27,36 \%$ en el año 2010 a $21,64 \%$ en el año 2014.

Este análisis impulsa un estudio profundizado acerca de la inversión del Estado en educación y la diferencia entre el costo de las escuelas privadas y las estatales.

La asignación de recursos o inversión puede realizarse de dos maneras: por el lado de la demanda o por el lado de la oferta.

La asignación por el lado de la demanda se refiere a la distribución de los recursos en forma directa por alumno (por ejemplo: vouchers). La asignación por el lado de la oferta implica que el Gobierno defina criterios administrativos para la dis- tribución de los distintos recursos (humanos, financieros, bienes y servicios). De la manera que opera el financiamiento se trata de financiar la demanda en el caso de la demanda por educación de gestión privada, mientras que en el caso de la gestión estatal se trata de subsidiar la oferta.

Los datos muestran que la inversión del Estado en ambos sectores de gestión crece según el ciclo del nivel educativo. Es así que la inversión estatal media por alumno en el sector de gestión estatal oscila entre $\$ 21.000$ y $\$ 29.000$ según el nivel educativo, y en el caso de privada varía entre $\$ 3.900$ y $\$ 8.000$.

La distribución de la inversión del Gobierno para los niveles de educación primario y secundario favorece a las comunas más vulnerables (4, 8 y 9) en ambos sectores de gestión, reflejando la distribución de la matrícula.

Dentro de cada sector de gestión, la dispersión en el gasto por alumno en las escuelas del nivel primario prueba la variabilidad existente entre escuelas. En el sector de gestión privada la diferencia es notoria.

Cuadro 2. Presupuesto ejecutado por el MEGC, 2010-2014 (en millones de pesos argentinos corrientes)

\begin{tabular}{|l|c|c|c|c|c|}
\hline & \multicolumn{5}{|c|}{ Año } \\
\hline & 2010 & 2011 & 2012 & 2013 & 2014 \\
\hline MEGC & 4.936 & 6.455 & 8.531 & 9.915 & 12.873 \\
\hline GC & 18.039 & 26.939 & 32.905 & 40.549 & 59.501 \\
\hline MEGC/GC (en \%) & 27,36 & 23,96 & 25,93 & 24,45 & 21,64 \\
\hline
\end{tabular}

Fuente: Elaboración propia con base en datos de S. Montoya (2015). 
Cuadro 3. Inversión estatal media por alumno según nivel educativo y sector de gestión. CABA ( 2013)

\begin{tabular}{|l|c|c|c|}
\hline \multicolumn{1}{|c|}{ Nivel } & Estatal & Privada & $\begin{array}{c}\text { Privada/Estatal } \\
\text { (en \% del gasto } \\
\text { estatal) }\end{array}$ \\
\hline Inicial & AR\$ 21.646 & AR\$ 3.991 & 18,4 \\
\hline Primario & AR\$ 24.795 & AR\$ 4.093 & 16,5 \\
\hline Secundario & AR\$ 28.937 & AR\$ 7.863 & 27,2 \\
\hline
\end{tabular}

Fuente: Elaboración propia con base en datos de S. Montoya (2015).

Gráfico 8. Distribución de la inversión del Gobierno según nivel de educación y sector de gestión, por comuna. CABA (2013)

Nivel primario
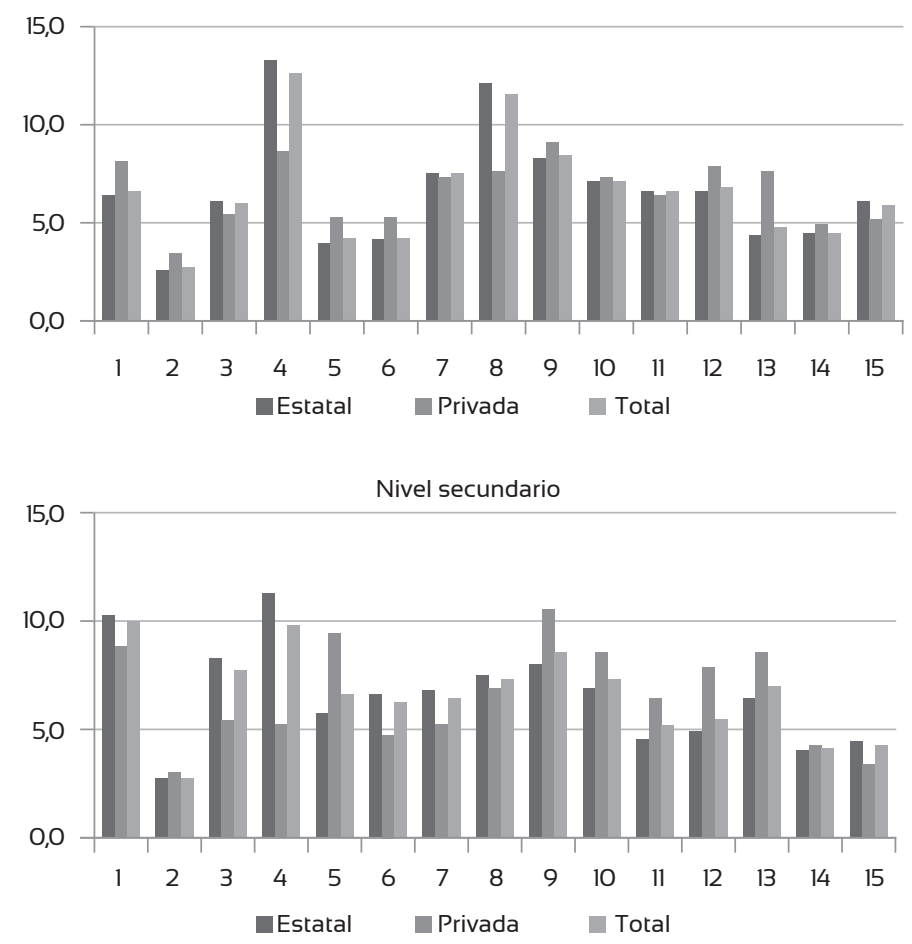

Fuente: Elaboración propia con base en datos de S. Montoya (2015). 
Gráfico 9. Dispersión en el gasto por alumno según sector de gestión para el nivel primario de educación. CABA (2013)

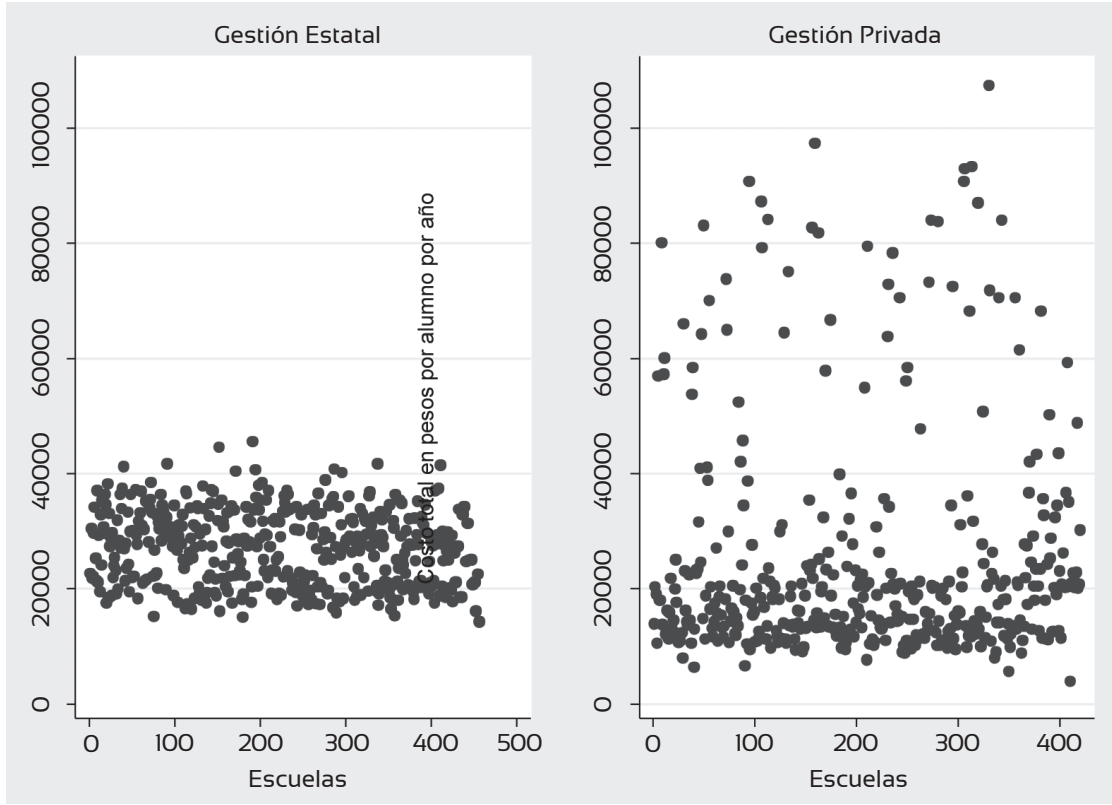

Fuente: Elaboración propia con base en datos de S. Montoya, (2015).

Podría concluirse entonces que la distribución de la inversión de la CABA en educación se realiza con miras a lograr una mayor equidad, favoreciendo a las zonas más vulnerables de la jurisdicción para poder así impulsar su mayor desarrollo.

\section{Eficiencia del sistema educativo}

Una manera de analizar la eficiencia en la Ciudad de Buenos Aires y aportar evidencia acerca de la misma en el marco del debate presentado al inicio de este documento es revisando el estudio sobre el IECEP y sus principales resultados (Montoya \& Volman, 2013). Este índice busca a través de variables de resultados de aprendizaje e indicadores de eficiencia interna estimar la calidad y equidad educativa de las escuelas secundarias de la Ciudad.

Debido a que los resultados de aprendizaje de los alumnos se encuentran influenciados por su contexto socioeconómico y esto se traduce en los valores que adopta el IECEP, se realizó un ajuste a dicho índice por nivel socioeconómico (IECEPa). Esto repercutió en diferencias entre los valores mínimos y máximos y en el valor promedio. El ajuste busca en definitiva presentar una medida representativa de los resultados sin un sesgo asociado al nivel socioeconómico. 
Partiendo del IECEP y del IECEPa, y de la inversión por escuela, se puede estimar la eficiencia del sistema educativo. Los resultados del estudio mencionado reflejan que la relación entre recursos y resultados del IECEP no es positiva. En las escuelas de gestión estatal, las de modalidad técnica obtienen resultados del índice superiores a escuelas de otras modalidades que cuentan con la misma cantidad de recursos. La misma relación no positiva existe entre recursos y el IECEPa, aunque en menor medida.

Gráfico 10. Relación costo/subsidio estatal por alumno y el IECEP y IECEPa
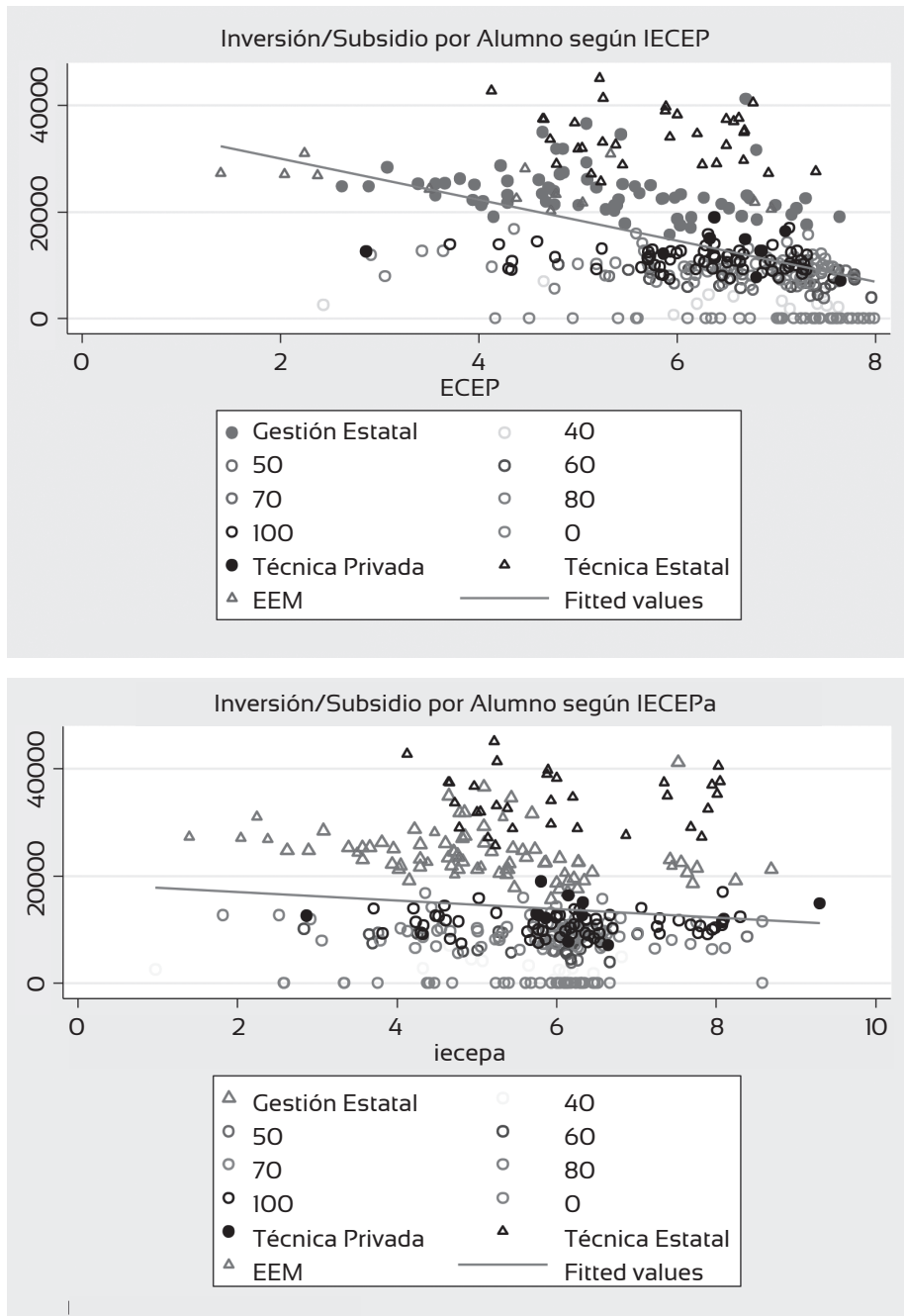

para el nivel secundario de educación (2013)

Fuente: Elaboración propia con base en datos de S. Montoya (2015). 
Gráfico 11. Relación entre el costo por alumno y el IECEP y el IECEPa para el nivel secundario de educación (2013)
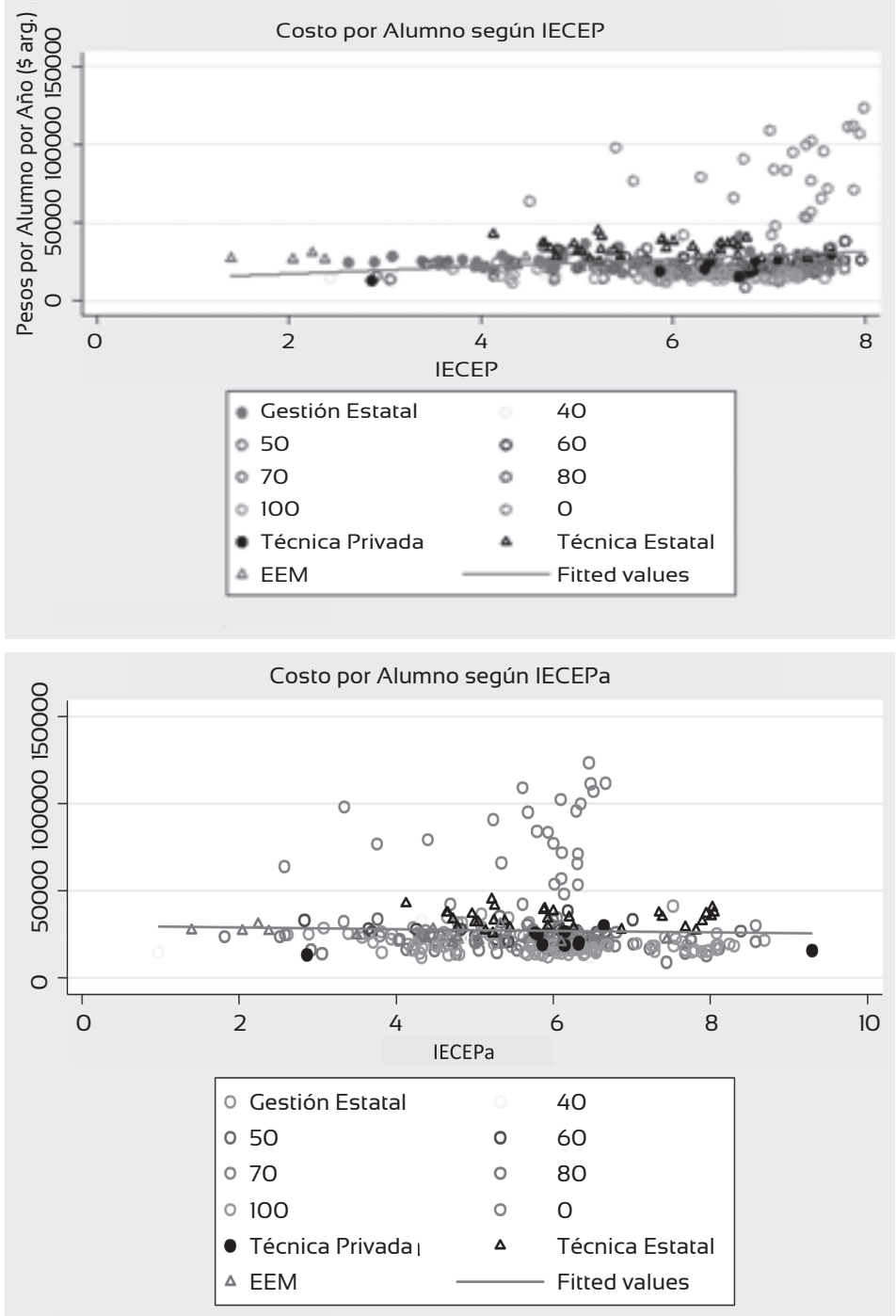

Fuente: Elaboración propia con base en datos de S. Montoya (2015).

Los resultados para las escuelas de gestión privada también prueban que mayores recursos no necesariamente implican mejores resultados.
Esto se puede observar en el gráfico 10, en el cual los triángulos rojos muestran escuelas de gestión estatal, los azules son escuelas de educación 
media y los negros escuelas técnicas estatales. Por otro lado, los puntos muestran las escuelas de gestión privada; se diferencian por color según porcentaje de aportes estatales recibidos, los negros representan a las escuelas técnicas privadas.

Manteniendo fijo el nivel de gasto por alumno, se puede observar la diferencia mencionada en los resultados del IECEP y IECEPa a través del valor que adoptan sobre el eje de las abscisas. Se puede percibir cómo mayores niveles de costo/subsidio estatal no necesariamente implican mejor calidad educativa.

Cuando se analiza el costo por alumno con el IECEP y el IECEPa los resultados nuevamente demuestran heterogeneidad. Escuelas con mismos costos por alumno que logran resultados diferentes en los índices, y escuelas que por el nivel de costo por alumno que manejan deberían presentar índices superiores a los que presentan. En otras palabras, no es posible aún hablar de eficiencia.

\section{Los resultados del ajuste de los microcostos por el IECEP}

Resulta inminente realizar un ajuste en los microcostos a través de un indicador de calidad educativa. Partiendo del análisis ya realizado, se ajustará el costo por alumno del sector de gestión estatal y del sector de gestión privada por el IECEP.

Para ello, se adopta la metodología utilizada por Pritchett y Aiyar (2014), fundada en preguntas como: ¿qué resultados de aprendizaje se obtendrían en cada sector de gestión con niveles equivalentes de gasto?, o ¿qué resultados de aprendizaje obtendrían las escuelas estatales con niveles de costos de escuelas privadas?

De esta manera también se puede estimar cuánto debería invertir el Estado en el sector de gestión estatal para alcanzar niveles de aprendizaje similares a los del sector privado.

Las variables que se utilizan en este análisis son entonces el costo por alumno y el IECEP. Las distribuciones de ambas variables se muestran en los gráficos 12 y 13 según sector de gestión. La función de densidad de la variable costo por alumno muestra dos distribuciones de cola derecha, mientras que las funciones de IECEP muestran distribuciones de cola izquierda. 
Gráfico 12. Funciones de densidad del costo por alumno para los sectores de gestión estatal y privada

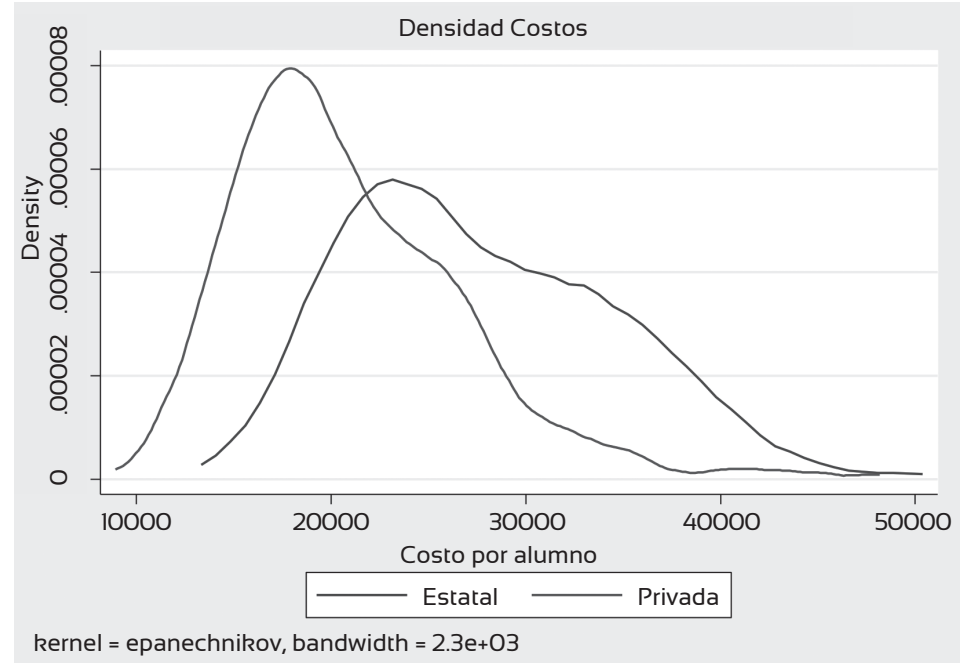

Fuente: Elaboración propia.

Gráfico 13. Funciones de densidad de la variable IECEP para los sectores de gestión estatal y privada

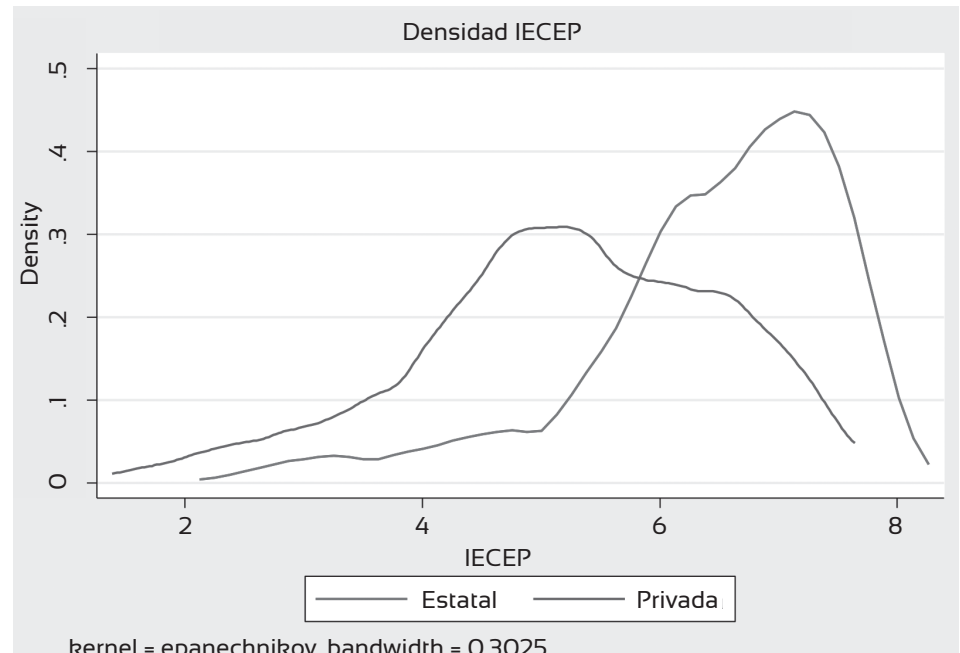

Fuente: Elaboración propia. 
La estimación se realiza a partir de los resultados de las regresiones simples entre el costo por alumno y el IECEP, cetaris paribus, para cada sector de gestión.

De esta manera, para cada sector de gestión se estimó:

$$
\operatorname{IECEP} P_{\text {gestión }}=\propto_{\text {gestión }}+\beta_{\text {gestion }} \text { costo al } ._{\text {gestión }}
$$

Los resultados de las regresiones simples arrojaron los siguientes valores:

\begin{tabular}{|l|c|c|}
\hline & Estatal & Privada \\
\hline Constante & 5,4899 & 5,5687 \\
\hline Coefic. costo por alumno & $-0,000009612$ & 0,00004174 \\
\hline
\end{tabular}

Y la media de cada variable adoptó los siguientes valores:

\begin{tabular}{|l|c|c|}
\hline & Estatal & Privada \\
\hline Media costo por alumno & 27707,54 & 20921,11 \\
\hline Media IECEP & 5,224 & 6,442 \\
\hline
\end{tabular}

Con los valores de $\alpha$ y $\beta$ se calculó el nivel previsto de IECEP del sector privado según el costo por alumno promedio del sector estatal:

$$
\begin{gathered}
\text { IECEP previsto } \text { privado }_{o}=\propto_{\text {privado }}+\beta_{\text {privado }} \times \text { costo al } l_{\text {estatal }} \\
\text { IECEP previsto }{ }_{\text {privado }}=5,5687+(0,00004174 \times 27707,54)=6,73
\end{gathered}
$$

El resultado se interpreta de la siguiente manera: para niveles de costo estatales por alumno $(\$ 27.707,54)$ se prevé que el sector de gestión privada alcance niveles de calidad educativa asociados a un IECEP de 6,73.

De la misma manera, se estimó el nivel previsto de IECEP del sector estatal para niveles de costo por alumno promedio del sector privado:

$$
\text { IECEP previsto } \text { estatal }=5,4899+(-0,000009612 \times 20921,11)=5,29
$$

Procediendo con el objetivo de estimar el nivel necesario de inversión del Gobierno en el sector de gestión estatal que permita niveles de calidad educativa correspondientes a los del sector de gestión privada se calcula: 


$$
\begin{gathered}
\text { Costo previsto al } l_{\text {estatal }}=\frac{I E C E P_{\text {privada }}-\alpha_{\text {estatal }}}{\beta_{\text {estatal }}} \\
\text { Costo previsto al } l_{\text {estatal }}=\frac{6,442-5,4899}{0,000009612}=99097
\end{gathered}
$$

Cuadro 4. Robustez del modelo de estimación de costo por alumno

\begin{tabular}{l|c|c|} 
& $\begin{array}{c}\text { Ganancia de aprendizaje } \\
\text { estimada por \$ adicional }\end{array}$ & $\begin{array}{c}\text { Doble costo para ganancia } \\
\text { de aprendizaje }\end{array}$ \\
\hline $\begin{array}{l}\beta_{\text {estatal }} \\
\text { \$ implicados en la } \\
\text { mejora en 0,1 puntos } \\
\text { del IECEP }\end{array}$ & 0,000009612 & 0,000004806 \\
\hline $\begin{array}{l}\text { estatal } \\
\text { Diferencia costo estatal - } \\
\text { costo privada (para IECEP } \\
=6,44)\end{array}$ & 5,4899 & 20807,32 \\
\hline
\end{tabular}

Fuente: Elaboración propia.

De esta manera, para que el sector de gestión estatal alcance niveles de IECEP asociados al sector de gestión privada, el Gobierno debería invertir $\$ 99.097$ por alumno, mientras que por alumno de gestión privada la inversión requerida es de $\$ 20.902$. Es decir que el costo por alumno estatal debería incrementarse en $\$ 71.389$.

Sin embargo, esta estimación presenta deficiencias por cuanto no considera los posibles efectos del contexto y del nivel socioeconómico del alumno característico de cada sector de gestión que pueden influir en los resultados y a la hora de explicar las mejoras en la calidad educativa. Por ello, se prueba la robustez del modelo disminuyendo el efecto del costo por alumno sobre los resultados. De esta manera, al disminuir el $\beta$, en este caso a la mitad, para alcanzar una mejora de 0,1 puntos en el IECEP será necesario el doble de pesos. Por otro lado, la diferencia entre el costo estatal y el costo privado por alumno se duplicó, aun aplicando la misma penalidad sobre el $\beta$ del sector de gestión privada (cuadro 4). 


\section{Control por nivel socioeconómico (NSE)}

Como se mencionó en un principio, uno de los ejes de la discusión entre educación de gestión estatal y privada es la segregación escolar: se suele asociar el sector de gestión estatal con un nivel socioeconómico bajo, mientras el sector privado se asocia a niveles socioeconómicos medio y alto. Esto es en definitiva una caracterización de la población que asiste a establecimientos educativos de cada sector de gestión, y una de las piezas faltantes en el modelo anterior.

De esta manera, el segundo ejercicio será ajustar los microcostos no solo por el IECEP sino también por alguna medida del nivel socioeconómico (NSE).

Un proxy del NSE que se suele utilizar es el nivel educativo de la madre. Al introducirlo en las regresiones lineales se obtienen coeficientes menores a los del modelo anterior.

\begin{tabular}{|l|c|c|}
\hline & Estatal & Privada \\
\hline Constante & $-0,40061847$ & 2,1492978 \\
\hline $\begin{array}{l}\text { Coefic. costo por } \\
\text { alumno }\end{array}$ & 0,000002773 & 0,000004297 \\
\hline
\end{tabular}

Nuevamente, estimamos el IECEP previsto para ambos sectores de gestión y se puede observar cómo impacta la introducción del nivel socioeconómico en los resultados:

IECEP $_{\text {previsto }}$ privado $=2,1492978+(0,000004297 \times 27707,54)=2,2$

IECEP previsto $_{\text {privado }}=-0,40061847+(0,000002773 \times 20921,11)=-0,34$

Para que un alumno del sector de gestión estatal alcance valores de IECEP asociados a la gestión privada, el costo por alumno asciende radicalmente a $\$ 2.467 .746$.

$$
\begin{gathered}
\text { Costo previsto } a_{\text {estatal }}=\frac{I E C E P_{\text {privada }}-\alpha_{\text {estatal }}}{\beta_{\text {estatal }}} \\
\text { Costo previsto al } \text { estatal }_{1}=\frac{6,442442-(-0,40061847)}{0,000002773}=2467746,29
\end{gathered}
$$


Se puede observar la repercusión y en cierta medida el impacto que el NSE de los alumnos tiene sobre el costo por alumno. Para alcanzar mayores niveles de calidad y equidad educativa, el costo por alumno del sector de gestión estatal, que se asocia a un nivel socioeconómico más bajo debido al menor valor promedio del nivel educativo alcanzado por la madre, debe ser mucho más alto. Ahora bien, el costo previsto por alumno privado asciende a \$999.102. En términos relativos la eficiencia de la gestión privada disminuiría casi a la mitad: mientras que con el ejercicio anterior la relación costo estatal/costo privada era de 4,76 , con la introducción del NSE la relación pasa a ser de 2,47.

Con el cálculo inicial, al no incluir los efectos del NSE y considerar características del alumno iguales entre los sectores de gestión, el costo por alumno estatal fue mucho más bajo que al considerar el impacto del NSE. En el segundo caso, el alto costo podría interpretarse como la inversión necesaria para solventar los efectos del NSE sobre los resultados de calidad y equidad educativa. En todo caso, cabe destacar la mejora en la posición del sector estatal con respecto al privado en el segundo ejercicio, lo que implica en cierta manera una disminución de la eficiencia relativa de la escuela privada con respecto a la escuela estatal.

\section{Conclusiones y lineamientos políticos}

La participación creciente del sector de gestión estatal en la última década en Argentina es foco de debate. ¿Es eficiente? ¿Cómo se relaciona con la equidad y calidad educativa?

Este trabajo intenta responder a ambas preguntas, con énfasis en la CABA.

A lo largo de este artículo se ha presentado evidencia acerca del estado de la educación de gestión privada en la Ciudad. Desde los aportes estatales a escuelas de gestión privada, hasta la inversión del Gobierno en escuelas de este sector de gestión se ha demostrado que el accionar en materia de distribución de recursos es de carácter progresivo y en pos de una mayor equidad educativa, al favorecer a las escuelas y comunas que se consideran más vulnerables según los indicadores sociales. Si es suficiente o no, no lo sabemos; pero sí se estaría realizando en la dirección correcta.

Una manera adicional de saber si es correcto o no, es mediante el análisis de la eficiencia.Los resultados reflejaron que no necesariamente una mayor cantidad de recursos implicó una mejoría de resultados, y que en muchos casos escuelas con el mismo nivel de recursos disponibles presentaron resultados disímiles.

Un primer análisis de los microcostos demostró que para que el sector de gestión estatal alcanzara niveles de calidad y equidad educativa 
similares a los que caracterizan al sector de gestión privada, el gasto por alumno debería aumentar considerablemente. Mientras un valor de IECEP de 6,442 se alcanza en el sector de gestión privada con un costo por alumno alrededor de $\$ 20.900$, en el sector de gestión estatal se alcanzaría con un costo por alumno de \$99.097. En líneas generales, se podría decir que el sector de gestión privada alcanza valores de IECEP Con menores niveles de gasto, lo que aludiría a mayores niveles de eficiencia con respecto al sector de gestión estatal.

Al incluir la variable educación de la madre como proxy del nivel socioeconómico, los resultados cambiaron considerablemente. Para que un alumno del sector de gestión estatal alcanzara valores de IECEP asociados a la gestión privada, el costo por alumno debería ser de $\$ 2.467 .746$. Se podría interpretar que parte del incremento en este costo se debe a la compensación por el NSE. Lo más interesante resulta al observar cómo la eficiencia relativa de la escuela privada sobre la estatal se reduce casi a la mitad con la inclusión del NSE en el modelo.

Además de esta diferencia de resultados de aprendizaje entre escuelas del sector de gestión privada y del sector de gestión estatal que aún es notoria, la variabilidad intrasector de gestión también se presenta como un factor para mejorar.

En conclusión, si bien las decisiones y el accionar de los hacedores de política parecerían estar orientados hacia alcanzar una mayor calidad, equidad y eficiencia educativa a través de políticas progresivas, no es posible hablar de eficiencia aún ni en el sector de gestión estatal ni en el sector de gestión privada, y lo que muchas veces se considera como mayor eficiencia del sector privado puede deberse simplemente a factores externos a la gestión misma y a "externalidades positivas" del contexto socioeconómico de la población que asiste a este tipo de establecimientos.

\section{Referencias}

Carnoy, M. \& McEwan, P. J. (1997). Is private education more effective and cost-effective than public? The case of Chile. Stanford University School of Education.

Banco Mundial. (1996-2013). Indicadores del Desarrollo Mundial. http://datos.bancomundial.org/indicador

Instituto Nacional de Estadísticas y Censos (Indec) (2010). Censo Nacional de Población, Hogares y Viviendas. Disponible en: http://www.censo2010.indec.gov.ar/ 
Jiménez, E., Lockheed, M. et al. (1995). Public and private secondary education in developing countries: a comparative study. World Bank Discussion Paper 309.

Montoya, S. et al. (2001). ¿Hacia dónde va el gasto público en educación? Logros y desafíos, 4. Una mirada comparativa: Argentina y Brasil. División de Desarrollo Social, Naciones Unidas y Cepal. Disponible en: http://repositorio.cepal.org/ bitstream/handle/11362/5996/ S01010070_es.pdf?sequence $=1$ Montoya, S. (2014). Subsidios a escuelas privadas: Análisis de los aportes de la Ciudad a las escuelas públicas de gestión privada para el nivel primario. Centro de Estudios de la Educación Argentina, Universidad de Belgrano. Disponible en: http://www.ub.edu. ar/centros_de_estudio/cea/lecturas_recomendadas/subsidios_ por_comuna_04ago2014.pdf

Montoya, S. \& Volman, V. (2014). ¿Cuáles son los determinantes de la calidad educativa en la Ciudad de Buenos Aires? Los índices de calidad y equidad porteños. Documento de trabajo, 44 de la Escuela de Economía Francisco Valsecchi. Facultad de Ciencias Económicas. Pontificia Universidad Católica Argentina. Disponible en: http://bibliotecadigital. uca.edu.ar/repositorio/investigacion/cuales-son-determinantescalidad.pdf
Montoya, S. (2015). La inversión invertida en la Ciudad de Buenos Aires: Análisis a partir de un sistema de costos por escuela. Proyecto Educar 2050. Disponible en: http://educar2050.org. ar/2015/marzo/Costos.pdf

Morduchowicz, A. (2000). La equidad del gasto educativo: viejas desigualdades, diferentes perspectivas. Revista Iberoamericana de Educación, 23.

Narodowski, M. \& Andrada, M. (2000). Segregación socioeconómica y regulaciones en el sistema educativo. Centro de Estudios para el Desarrollo Institucional. Fundación Gobierno y Sociedad. Documento 36. Disponible en: http://faculty.udesa.edu.ar/tommasi/cedi/dts/dt36.pdf

Pritchett, L. \& Aiyar, Y. (2014). Value subtraction in public sector production: Accounting versus economic cost of primary schooling in India. CGD Working Paper 391. Washington, DC: Center for Global Development. Disponible en: http://www.cgdev.org/publication/value-subtraction-publicsector-productionaccountingversus-economic-cost-primary

Relevamientos Anuales, (1996-2013). Dirección Nacional de Información y Evaluación de la Calidad Educativa (DINIECE), Ministerio de Educación de la Nación Argentina. Disponible en: http://portales.educacion.gov.ar/diniece/ 
Somers, M. A. et al. (2011). How effective are private schools in Latin America? Comparative Education Review, 48 (01). University of Chicago Press on behalf of the Comparative and International Education Society. Disponible en: http://www. jstor.org/stable/10.1086/379841 